\title{
Structural and Volumetric Brain MRI Findings in Mild Traumatic Brain Injury
}

\author{
(D).B. Patel, (D) S.H. Wilson, (D)T.R. Oakes, (D) P. Santhanam, and (D).K. Weaver
}

\begin{abstract}
BACKGROUND AND PURPOSE: Routine MR imaging findings are frequently normal following mild traumatic brain injury and have a limited role in diagnosis and management. Advanced MR imaging can assist in detecting pathology and prognostication but is not readily available outside research settings. However, 3D isotropic sequences with $\sim 1-\mathrm{mm}^{3}$ voxel size are available on community MR imaging scanners. Using such sequences, we compared radiologists' findings and quantified regional brain volumes between a mild traumatic brain injury cohort and non-brain-injured controls to describe structural imaging findings associated with mild traumatic brain injury.
\end{abstract}

MATERIALS AND METHODS: Seventy-one military personnel with persistent symptoms and 75 controls underwent $3 T$ MR imaging. Three neuroradiologists interpreted the scans using common data elements. FreeSurfer was used to quantify regional gray and white matter volumes.

RESULTS: WM hyperintensities were seen in $81 \%$ of the brain-injured group versus $60 \%$ of healthy controls. The odds of $\geq 1$ WM hyperintensity in the brain-injured group was about 3.5 times the odds for healthy controls $(95 \% \mathrm{Cl}, 1.58-7.72 ; P=.002)$ after adjustment for age. A frontal lobe-only distribution of WM hyperintensities was more commonly seen in the mild traumatic brain injury cohort. Furthermore, 7 gray matter, 1 white matter, and 2 subcortical gray matter regions demonstrated decreased volumes in the brain-injured group after multiple-comparison correction. The mild traumatic brain injury cohort showed regional parenchymal volume loss.

CONCLUSIONS: White matter findings are nonspecific and therefore a clinical challenge. Our results suggest that prior trauma should be considered in the differential diagnosis of multifocal white matter abnormalities with a clinical history of mild traumatic brain injury, particularly when a frontal predilection is observed.

ABBREVIATIONS: $\mathrm{DAI}=$ diffuse axonal injury; $\mathrm{mTBI}=$ mild traumatic brain injury; $\mathrm{TAI}=$ traumatic axonal injury; $\mathrm{TBI}=$ traumatic brain injury; WMH $=$ white matter hyperintensity

M any patients experiencing mild traumatic brain injury (mTBI) report symptom resolution and return to baseline function within 3 months. A subset (3\%-53\%) develop postconcussive symptoms that persist months to years postinjury, ${ }^{1-3}$

Received August 21, 2019; accepted after revision October 16.

From Lovelace Biomedical Research (J.B.P., T.R.O., P.S.), Albuquerque, New Mexico; VA Maryland Health Care System (J.B.P.), Baltimore, Maryland; Emmes (S.H.W.) Rockville, Maryland; Division of Hyperbaric Medicine (L.K.W.), Intermountain Medical Center, Murray, Utah, and Intermountain LDS Hospital, Salt Lake City, Utah; University of Utah School of Medicine (L.K.W.), Salt Lake City, Utah; and University of Wisconsin-Madison (T.R.O.), Madison, Wisconsin.

This work was supported by the United States Army Medical Research and Materiel Command Contract No. W81XWH-15-D-0039-0003.

The views, opinions and/or findings contained in this report are those of the authors and should not be construed as an official Department of the Army, Veterans Affairs, or US government position, policy, or decision unless so designated by other documentation. In the conduct of research in which humans are the subjects, the investigators adhered to the policies regarding the protection of human subjects as prescribed by Code of Federal Regulations Title 45, Volume 1, Part 46; Title 32, Chapter 1, Part 219; and Title 21, Chapter 1, Part 50 (Protection of Human Subjects). including dizziness, headache, cognitive deficits, sleep disturbances, tinnitus, visual symptoms, and behavioral/affective changes. ${ }^{1,4}$ Because symptoms are nonspecific to $\mathrm{mTBI}$ and routine clinical neuroimaging findings are frequently normal, identifying imaging correlates and biomarkers of postconcussive syndrome is of clinical importance.

MR imaging is the appropriate technique for patients with subacute or chronic mTBI with cognitive or neurologic deficits, ${ }^{5}$ but routine clinical MR imaging may have limited utility for diagnosis and management. ${ }^{6}$ Advanced MR imaging sequences obtained at 3T field strength demonstrate changes not

Please address correspondence to Lindell K. Weaver, MD, FACP, FCCP, FCCM, FUHM, Intermountain LDS Hospital Hyperbaric Medicine, 8th Ave and C St, Salt Lake City, UT 84143; e-mail: lindell.weaver@imail.org

\footnotetext{
--.- Indicates open access to non-subscribers at www.ajnr.org

三 Indicates article with supplemental on-line table.

http://dx.doi.org/10.3174/ajnr.A6346
} 
typically observable in routine clinical MR imaging. DTI, MR spectroscopy, fMRI, and perfusion techniques assist in pathology detection and may provide improved diagnostic and prognostic indicators; 6 however, postacquisition processing is required, group-based differences can be difficult to apply to individual patients, and such techniques are not readily available in community settings. High-resolution structural imaging (3D sequences with isotropic $1-\mathrm{mm}^{3}$ voxels that can be reconstructed in all planes) can be acquired on routine clinical scanners without time-intensive postprocessing. High-resolution MR imaging scans, recommended by the Defense Centers of Excellence, ${ }^{7}$ are regarded as best practice for traumatic brain injury (TBI).

A challenge in the interpretation of such structural imaging is determining the importance of imaging findings identified more easily at higher resolution and field strengths. ${ }^{8}$ White matter hyperintensities (WMHs), dilated perivascular spaces, pineal gland cysts, and pituitary gland abnormalities have been identified in both mTBI and healthy cohorts, ${ }^{9,10}$ and the imaging manifestation of multifocal T2 and FLAIR hyperintensity is visible across conditions including demyelination, inflammation, chronic small vessel ischemia, normal aging, migraine headaches, and moderate/severe TBI. Recent studies in military populations have demonstrated similar rates of WMH in mTBI and non-head trauma groups (Tate et al, ${ }^{11} 41 \%$ mTBI versus $49 \%$ orthopedically injured versus $29 \%$ posttraumatic stress disorder only; and Riedy et al, ${ }^{9} 51.8 \%$ mTBI versus $38.1 \%$ healthy controls).

Established software packages (eg, FreeSurfer; http://surfer. nmr.mgh.harvard.edu) ${ }^{12}$ can quantify regional white matter and gray matter volumes, with longitudinal decreases in brain volumes following $\mathrm{mTBI}^{13}$ in the anterior cingulate white matter bilaterally, left cingulate gyrus isthmus white matter, and right precuneal gray matter. Many studies report group differences, but large between- and within-group variance limits interpretation for individual patient diagnosis.

In this article, we describe the clinical interpretation and volumetric analysis of MR imaging scans acquired during the BIMA (Brain Injury and Mechanisms of Action of $\mathrm{HBO} 2$ for Persistent Post-Concussive Symptoms After Mild Traumatic Brain Injury) study, ${ }^{14}$ a randomized, double-blind study of hyperbaric oxygen in US military personnel with persistent postconcussive symptoms after mTBI (clinicaltrials.gov: NCT01611194). A complementary observational study, NORMAL (Development of Normative Datasets for Assessments Planned for Use in Patients With Mild Traumatic Brain Injury), ${ }^{15}$ evaluated nonbrain-injured volunteers using the same imaging protocol on the same scanner (clinicaltrials.gov: NCT01925963). We compare baseline structural imaging findings, WMH burden, and segmented white and gray matter volumes between mTBI and healthy control groups. We also test imaging findings for correlation with injury characteristics and clinical outcome and compare the results with those of other military mTBI cohorts.

\section{MATERIALS AND METHODS Study Populations}

BIMA enrolled 71 military personnel (70 with MR imaging data are included in the analysis) with $\geq 3$ persistent symptoms from active duty mTBI 3 months to 5 years before enrollment. Mild
TBI was assessed by structured interview ${ }^{16}$ and included loss of consciousness of $\leq 30$ minutes, altered consciousness of $\leq 24$ hours, or posttraumatic amnesia of $\leq 1$ day. Seventy participants with complete MR imaging data are included in this report. NORMAL enrolled 75 participants; prior stroke, infection, therapeutic ionizing radiation exposure, neurologic disorder, chronic migraine headaches, drug/alcohol abuse, and diabetes mellitus were exclusionary. Primary study results are published elsewhere. $^{14,15}$ The United States Army Medical Research and Materiel Command institutional review board approved the studies, and participants provided written informed consent.

\section{MR Imaging Protocol}

Participants underwent 3T structural MR imaging (Achieva, Software Release 3.2; Philips Healthcare, Best, the Netherlands; 32-channel sensitivity encoding head coil) without the use of gadolinium at Evans Army Community Hospital, a 92-bed community hospital. Three trained technologists performed the imaging protocol. Although there was no on-site MR imaging physicist, experienced MR imaging researchers made frequent visits for training and setup, quality control, and troubleshooting. The complete MR imaging acquisition protocol is provided elsewhere. $^{17}$

Participants underwent an initial quick-scan protocol consisting of rapid T2 and FLAIR sequences, which were loaded onto the hospital PACS system and interpreted at the time of imaging by a hospital radiologist to screen for major abnormalities and document the MR imaging in the military medical record.

Structural sequences were acquired at a spatial resolution as high as possible while maintaining good signal quality. Anatomic images included isotropic 3D T1-weighted, T2-weighted, T2 FLAIR, and 2D $\mathrm{T}^{*}$-weighted. The full MR imaging protocol required approximately 2 hours. Herein we report anatomic MR imaging and FreeSurfer results. DICOM files were conditioned by using the Radiological Society of North America Clinical Trials Processor and distributed to clinical specialists for processing (https://www.rsna.org/research/imaging-research-tools).

\section{MR Imaging Clinical Interpretation}

Each scan was assigned to 2 of 3 board-certified neuroradiologists for independent review. In the event of disagreement on any finding, all 3 reviewed and discussed the findings to arrive at a consensus on the final scoring. The radiologists viewed DICOM images via OsiriX Imaging Software (http://www.osirix-viewer. com), ${ }^{18}$ allowing real-time generation of MPR and MIP images and synchronizing the spatial location between series and across time points. These features were useful in evaluating small foci of white matter signal abnormality.

Interpretations were standardized using a Web-based case report form based on the National Institute of Neurological Disorders and Stroke neuroradiologic TBI common data elements (QuesGen Systems, Burlingame, California). ${ }^{19}$ The forms triggered external clinical referral in the event of critical findings.

Each neuroradiologist manually enumerated, annotated, and saved all relevant imaging findings using single-point ROIs and completed a series of electronic interpretation forms. We specifically evaluated and scored the following items: ventricular

AJNR Am J Neuroradiol 41:92-99 Jan 2020 www.ajnr.org 
enlargement, asymmetric ventricles, arachnoid cyst, possible diffuse axonal injury (DAI)/traumatic axonal injury (TAI), contusion, gliosis, microhemorrhage, intracerebral hemorrhage, brain atrophy, encephalomalacia, dilated perivascular spaces, cavum septum, pituitary abnormality, pineal cyst, sinus disease, mastoid fluid, lymphadenopathy, ischemia/infarction, intraventricular hemorrhage, epidural hematoma, subdural hematoma, subarachnoid hemorrhage, and edema.

The broad category of T2 and FLAIR white matter signal changes was classified as DAI/TAI when there was increased T2 and FLAIR white matter signal in a pattern and distribution that could be consistent with prior traumatic injury. ${ }^{9,20}$ Possible DAI/ TAI lesions were typically identified in the subcortical white matter often involving the frontal or parietal lobes; however, we also included periventricular and deep white matter foci in this classification. Other definitions included the following:

- Encephalomalacia-focal cortical volume loss

- Dilated perivascular spaces-longitudinally oriented cystic spaces nearly isointense to CSF on the T1, T2, and FLAIR sequences

- Gliosis - an area of T2 FLAIR white matter signal exceeding 3 $\mathrm{mm}$ with morphology atypical for DAI/TAI or in an area of encephalomalacia.

The terms DAI/TAI, white matter T2 FLAIR hyperintensities, $\mathrm{WMH}$, and white matter lesion burden are used interchangeably. For discrepant WMH counts, the larger number was reported.

\section{Quantified Volumetric Analysis}

Cortical and subcortical segmentation and volumetric quantification of the 3D T1-weighted sequence were performed using FreeSurfer (Version 5.3). Processing included removal of nonbrain tissue, ${ }^{21}$ automated Talairach transformation, segmenting of subcortical white matter and deep gray matter structures, ${ }^{12,22}$ intensity normalization, ${ }^{23}$ gray matter/white matter boundary tessellation, automated topology correction, ${ }^{24,25}$ and surface deformation following intensity gradients along the gray/white and gray/CSF borders. $^{26-28}$ Segmentations were checked to verify the automated reconstruction, and manual interventions corrected small processing defects and any segmentation inaccuracies due to the presence of lesions.

\section{Statistical Analyses}

Continuous outcomes are summarized using means and SDs, whereas discrete outcomes are summarized using counts and percentages. Statistical models include adjustment for age at study enrollment.

Baseline demographics are presented by study population with between-group differences tested using a Wilcoxon rank sum or $\chi^{2}$ test. Baseline characteristics were tested in the mTBI cohort between those with zero versus $\geq 1 \mathrm{WMH}$ using logistic regression.

Baseline MR imaging findings are presented when prevalence in either study group was $>3 \%$. Total and regional WMH distributions are summarized by the number of WMHs and by the prevalence of $\geq 1 \mathrm{WMH}$ with group differences, tested using logistic regression. Regional WMH patterns are displayed graphically using bar charts and were tested using the Fisher exact test.

Given the potential effect of sex on WMH burden, lesion prevalence was analyzed by sex for a subset of the healthy controls, and a sensitivity analysis was performed testing betweenstudy group WMH differences excluding women. We performed an exploratory analysis to try to understand more about participants with mTBI with outlier WMH counts (defined as any WMH count of $>1.5$, with interquartile ranges above the third quartile) and the following characteristics: TBI symptom duration ( 3 months to 5 years versus $1-5$ years postinjury), number of injuries (1 versus multiple), type of injuries (lifetime history of blast versus blunt force versus a combination), baseline posttraumatic stress disorder diagnosis, number of deployments, and military tenure. For characteristics in which visual inspection of summary statistics indicated a potential trend, logistic regression models were fit to model the probability of WMH outliers predicted by the characteristic and adjusted for age.

FreeSurfer regional volumes, expressed as a percentage of total intracranial volume, are presented by hemisphere, parcellation type (cortical gray matter, subcortical gray matter, white matter), and study group. ROI-specific between-group differences were tested using general linear models with $P$ values adjusted using the Holm-Bonferroni ${ }^{29}$ procedure applied within parcellation type. Heat maps display group differences by ROI.

The relationship between regional FreeSurfer volume and WMH burden, defined as total or frontal $\mathrm{WMH}$ of 0 vs $\geq 1$, was investigated by fitting general linear models of each regional volume with fixed effects of WMH burden and age. Models were fit separately for each study population and limited to those regions indicating a difference in volume between the mTBI and healthy control populations.

Analysis was performed using SAS 9.4 (SAS Institute, Cary, North Carolina), and statistical testing was performed at the $\alpha=$ .05 level unadjusted for multiple comparisons unless otherwise noted.

\section{RESULTS}

BIMA participants were younger (BIMA mean age, $33 \pm 7$ years; range, 21-53 years; NORMAL mean age, $39 \pm 13$ years; range, 18-65 years; $P=.008$ ), and more participants were male than healthy control participants (BIMA, 99\% male; NORMAL, 77\% male; $P<.001)$. BIMA recruited nearly all active duty military (97\%) compared with NORMAL (1\%). In BIMA, time since injury ranged from 4 to 60 months (mean, $25.6 \pm 16.2$ months), and injury types included a history of blast injuries $(32 \%, n=23)$, blunt force injuries $(20 \%, n=14)$, and a combination of blast/ blunt force injuries $(48 \%, n=34)$.

\section{Structural Findings}

The most common findings in the mTBI study group were DAI/ TAI, sinus disease, cavum septum, pineal cysts, dilated perivascular spaces, and brain atrophy (Table 1$)$. Only DAI/TAI $(n=56$, $80 \%$ BIMA versus $n=45$, 61\% NORMAL; OR $=3.06$; $95 \% \mathrm{CI}$, $1.40-6.69 ; P=.005)$ and brain atrophy $(n=22,31 \%$ BIMA versus $n=10,14 \%$ NORMAL; OR $=3.00 ; 95 \%$ CI, $1.23-7.30 ; P=.02)$ differed between groups. Incidental findings referred for clinical 


\begin{tabular}{|c|c|c|c|c|c|c|}
\hline & \multicolumn{2}{|c|}{ BIMA $(n=70)$ (No.) (\%) } & \multicolumn{2}{|c|}{ NORMAL $(n=75)$ (No.) (\%) } & \multirow[b]{2}{*}{ Odds Ratio $(95 \% \mathrm{Cl})^{\mathrm{a}}$} & \multirow[b]{2}{*}{$P$ Value } \\
\hline & $\mathrm{N}^{\mathrm{a}}$ & (No.) (\%) & $N^{a}$ & (No.) (\%) & & \\
\hline Arachnoid cysts & 70 & $6(8.6)$ & 70 & $3(4.3)$ & $1.74(0.41-7.37)$ & .45 \\
\hline Asymmetric ventricles & 70 & $14(20.0)$ & 70 & 8 (11.4) & $2.75(0.94-8.03)$ & .06 \\
\hline Brain atrophy & 70 & $22(31.4)$ & 71 & $10(14.1)$ & $3.00(1.23-7.30)$ & $.02^{\mathrm{c}}$ \\
\hline Cavum septum & 70 & $34(48.6)$ & 70 & $32(45.7)$ & $1.32(0.66-2.67)$ & .43 \\
\hline Developmental venous anomalies & 70 & $3(4.3)$ & 71 & $4(5.6)$ & $0.72(0.15-3.49)$ & .68 \\
\hline DAl/TAI & 70 & $56(80.0)$ & 74 & $45(60.8)$ & $3.06(1.40-6.69)$ & $.005^{\mathrm{c}}$ \\
\hline Dilated perivascular spaces & 70 & $33(47.1)$ & 72 & $34(47.2)$ & $1.06(0.53-2.12)$ & .86 \\
\hline Lymph nodes & 70 & 11 (15.7) & 71 & $7(9.9)$ & $1.51(0.50-4.55)$ & .47 \\
\hline Mastoid fluid & 70 & $6(8.6)$ & 70 & $1(1.4)$ & $6.44(0.70-59.08)$ & .10 \\
\hline Microhemorrhages & 70 & $3(4.3)$ & 70 & $0(0.0)$ & NA & NA \\
\hline Pineal cysts & 70 & $32(45.7)$ & 70 & $31(44.3)$ & $1.33(0.65-2.71)$ & .43 \\
\hline Pituitary abnormalities & 70 & 9 (12.9) & 71 & $13(18.3)$ & $1.15(0.40-3.33)$ & .80 \\
\hline Sinus disease & 70 & $35(50.0)$ & 71 & $38(53.5)$ & $0.95(0.48-1.89)$ & .89 \\
\hline
\end{tabular}

Note:-NA indicates not applicable.

${ }^{a}$ Number nonmissing for the denominator of the percentage calculation.

${ }^{b}$ Results obtained from logistic regression models of the probability of MR imaging findings adjusted for age.

${ }^{c} p<0.05$.

Table 2: Total and regional WMH distribution by study group

\begin{tabular}{|c|c|c|c|c|c|c|c|c|}
\hline & \multicolumn{3}{|c|}{ BIMA $(n=70)$} & \multicolumn{3}{|c|}{ NORMAL $(n=75)$} & \multirow[b]{2}{*}{$\begin{array}{l}\text { Odds Ratio } \\
(95 \% \mathrm{Cl})^{\mathrm{a}}\end{array}$} & \multirow[b]{2}{*}{$\begin{array}{c}P \\
\text { Value }^{\mathrm{a}}\end{array}$} \\
\hline & $\begin{array}{c}\text { No. (\%) } \\
\text { With } \geq 1 \\
\text { WMH }\end{array}$ & $\begin{array}{c}\text { Mean WMH } \\
\text { Count (SD) }\end{array}$ & $\begin{array}{l}\text { Median WMH } \\
\text { Count (Range) }\end{array}$ & $\begin{array}{c}\text { No. (\%) } \\
\text { With } \geq 1 \\
\text { WMH }\end{array}$ & $\begin{array}{c}\text { Mean WMH } \\
\text { Count (SD) }\end{array}$ & $\begin{array}{l}\text { Median WMH } \\
\text { Count (Range) }\end{array}$ & & \\
\hline Total WMH & $57(81.4)$ & $8.6(17.9)$ & $3(0-108)$ & $45(60.0)$ & $8.5(18.0)$ & $1(0-83)$ & 3.49 (1.58-7.72) & $.002^{\mathrm{b}}$ \\
\hline \multicolumn{9}{|l|}{ Regional WMH } \\
\hline Frontal & $52(74.3)$ & $6.3(13.0)$ & $2(0-69)$ & $44(58.7)$ & $6.0(13.3)$ & $1(0-74)$ & $2.43(1.16-5.08)$ & $.02^{\mathrm{b}}$ \\
\hline Parietal & $22(31.4)$ & $1.2(3.9)$ & $0(0-29)$ & $21(28.0)$ & $1.6(4.5)$ & $0(0-29)$ & $1.51(0.70-3.28)$ & .30 \\
\hline Temporal & $16(22.9)$ & $0.7(1.9)$ & $0(0-10)$ & $15(20.0)$ & $0.5(1.2)$ & $0(0-6)$ & $1.46(0.62-3.45)$ & .39 \\
\hline Occipital & $2(2.9)$ & $0.0(0.2)$ & $0(0-1)$ & $8(10.7)$ & $0.2(0.5)$ & $0(0-2)$ & $0.33(0.06-1.77)$ & .20 \\
\hline Cerebellum & $1(1.4)$ & $0.0(0.1)$ & $0(0-1)$ & $0(0.0)$ & $0.0(0.0)$ & $0(0-0)$ & NA & NA \\
\hline $\begin{array}{l}\text { Corpus } \\
\text { callosum } \\
\text { genu }\end{array}$ & $2(2.9)$ & $0.1(0.4)$ & $0(0-3)$ & $2(2.7)$ & $0.1(0.3)$ & $0(0-2)$ & $0.93(0.12-6.93)$ & .94 \\
\hline $\begin{array}{l}\text { Corpus } \\
\text { callosum } \\
\text { body }\end{array}$ & $8(11.4)$ & $0.1(0.4)$ & $0(0-2)$ & $5(6.7)$ & $0.1(0.5)$ & $0(0-3)$ & $2.12(0.6-7.51)$ & .25 \\
\hline $\begin{array}{l}\text { Corpus } \\
\text { callosum } \\
\text { splenium }\end{array}$ & $2(2.9)$ & $0.0(0.2)$ & $0(0-1)$ & $1(1.3)$ & $0.0(0.1)$ & $0(0-1)$ & 8.39 (0.29-242.91) & .22 \\
\hline Midbrain & $1(1.4)$ & $0.0(0.1)$ & $0(0-1)$ & $0(0.0)$ & $0.0(0.0)$ & $0(0-0)$ & NA & NA \\
\hline Pons & $0(0.0)$ & $0.0(0.0)$ & $0(0-0)$ & $1(1.3)^{\prime}$ & $0.0(0.2)$ & $0(0-2)$ & NA & NA \\
\hline Medulla & $0(0.0)$ & $0.0(0.0)$ & $0(0-0)$ & $0(0.0)$ & $0.0(0.0)$ & $0(0-0)$ & NA & NA \\
\hline
\end{tabular}

Note:-NA indicates not applicable.

${ }^{a}$ Results obtained from logistic regression models of the probability of $\geq 1 \mathrm{WMH}$ adjusted for age

${ }^{b} P<0.05$.

evaluation included the following: possible jugular foramen thrombus, maxillary bone lesion, pineal mass, possible demyelination, pituitary mass, and incidental intracranial aneurysms.

\section{WMH Findings}

We observed $\geq 1 \mathrm{WMH}$ in 57 (81\%) of the mTBI group versus 45 $(60 \%)$ in the healthy controls (Table 2). Logistic regression models were fit to model the probability of lesion burden (defined as $\geq 1 \mathrm{WMH}$ ) and included an effect for study (BIMA versus NORMAL) to test differences in lesion burden between the mTBI and healthy controls populations and a covariate for age. The odds of $\geq 1 \mathrm{WMH}$ in the mTBI group was about 3.5 times the odds for the healthy controls (95\% CI, 1.58-7.72; $P=.002$ ) after adjustment for age (odds ratio without age $=2.92$; $95 \%$ CI, 1.37 -
6.25; $P=.006)$. Similar findings were seen for frontal lobe WMH $(\mathrm{OR}=2.43$; 95\% CI, 1.16-5.08; $P=.02)$. Median total WMHs in the mTBI and healthy controls groups were 3 (range, $0-108$ ) and 1 (range, 0-83), respectively. Increasing white matter lesion burden in the mTBI-versus-healthy controls cohort was not observed in other regions (Table 2).

The most common lobar involvement pattern in the mTBI group was frontal-only $(n=26,37 \%)$ versus no $\mathrm{WMH}(n=30$, $40 \%$ ) in the healthy controls (Fig 1). Differences in patterns approached but did not reach significance $(P=.08)$.

Seventy-one percent $(n=12)$ of the female healthy controls had $\geq 1 \mathrm{WMH}$ versus only $39 \%$ of the male subgroup $(\mathrm{OR}=4.2$; 95\% CI, 0.96-18.42; $P=.06$ ). Results of this subanalysis should be interpreted with caution, given small within-group sample sizes. 


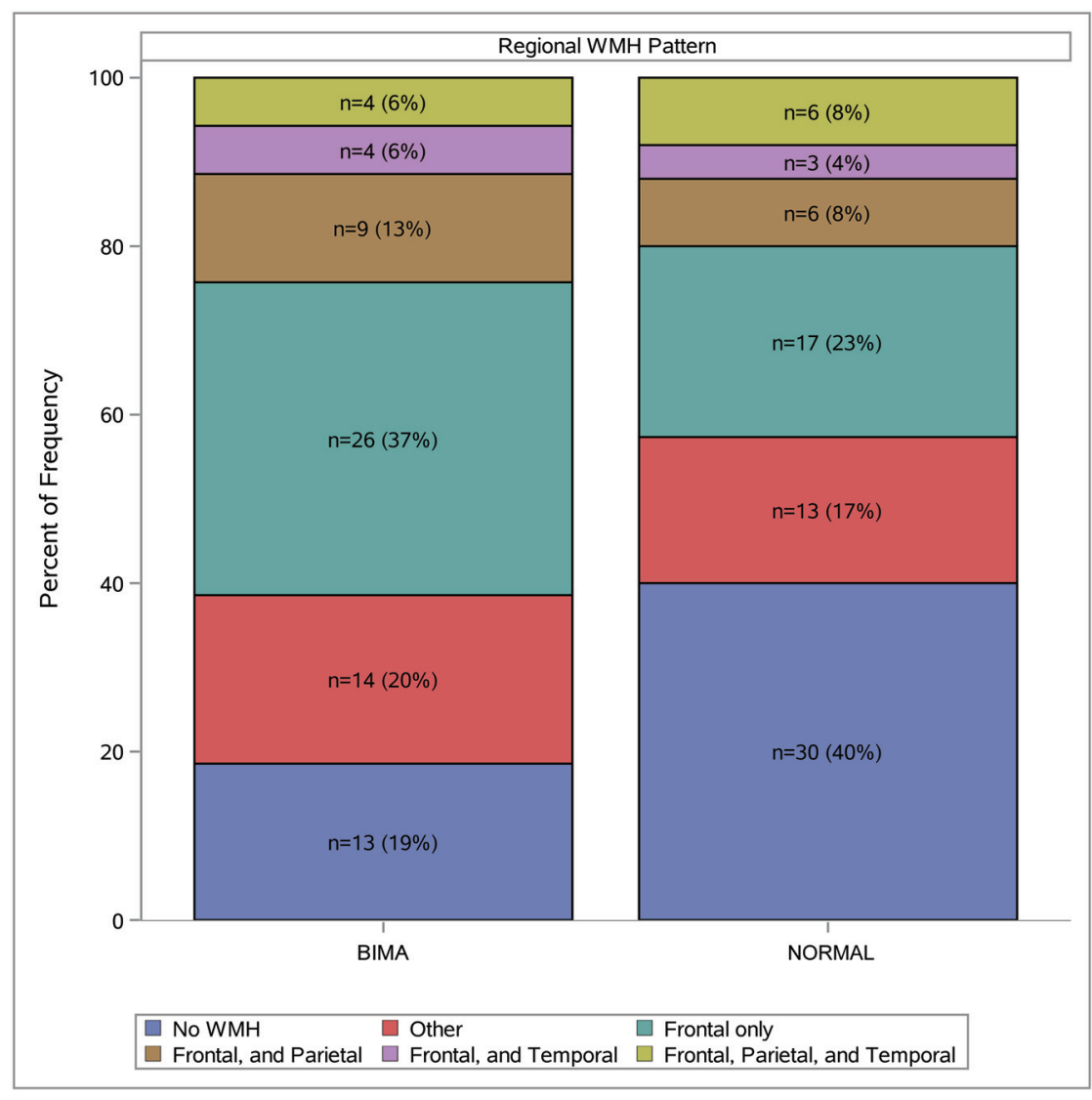

FIG 1. WMH pattern by study group. Distributions of regional WMH patterns are presented by study population. Patterns are classified by the presence of $\geq 1 \mathrm{WMH}$ in each region (eg, in BIMA $n=4,6 \%$ of participants were observed to have $\geq 1 \mathrm{WMH}$ in the frontal, parietal, and temporal regions). The most commonly observed patterns are presented in this figure; less commonly observed patterns are classified as "Other."

Results of the sensitivity analysis of WMH prevalence excluding women were similar to primary analysis results; 1 difference was that the OR for $\geq 1$ corpus callosum body WMH increased in magnitude and became significant $(\mathrm{OR}=8.17$; 95\% CI, 1.07$62.44 ; P=.04$; other data not shown).

No significant differences were detected between higher $(\geq 1)$ versus lower (zero) WMH burden for any baseline characteristics in the mTBI cohort, including age, military tenure, number of deployments, history of blast injury, time from injury (duration of postconcussive symptoms), or baseline composite clinical outcome scores ${ }^{30}$ (data not shown).

Exploratory analysis of participants with mTBI who had an outlier WMH count $(n=7)$ versus those without $(n=63)$ revealed a potential trend for longer military service (mean for $\mathrm{WMH}$ outliers = 16 years; $95 \% \mathrm{CI}, 12-20$ years; mean for $\mathrm{WMH}$ nonoutliers $=10$ years; 95\% CI, 9-12 years) and number of deployments (deployments for WMH outliers $=4.6$; 95\% CI, 2.8-6.3; for $\mathrm{WMH}$ nonoutliers $=2.7 ; 95 \% \mathrm{CI}, 2.2-3.4)$. However, after adjusting for age, these associations were not statistically significant.

\section{Volumetric Comparisons}

Regional volumetric analysis revealed smaller mean volumes in the mTBI-versus-healthy controls cohorts. We identified significant (adjusted) between-group volume differences in the left insula, rostral anterior cingulate cortex, and trans-verse temporal gyri, as well as the right lateral and medial orbitofrontal cortices and postcentral and precentral gyri of the 68 regional cortical gray matter comparisons, in the left transverse temporal gyrus of the 68 regional white matter comparisons, and in the left cerebellum cortical and right thalamus regions of the 28 subcortical gray matter comparisons (Fig 2 and On-line Table).

In the mTBI population, mean regional FreeSurfer volumes for all regions investigated were smaller among those with $\geq 1 \mathrm{WMH}$ versus those with no WMH. No clear pattern across regions was identified in the healthy controls population. However, in general linear models adjusted for age, the only significant finding was in the healthy controls population, in which an increase was observed in the right lateral orbitofrontal volume in those with $\geq 1 \mathrm{WMH}$ (adjusted mean $=0.51 ; 95 \% \mathrm{CI}, 0.45-$ 0.50) compared with no WMH (adjusted mean $=0.4 ; 95 \%$ CI, 0.490.53) $(P=.04)$. Similar findings were observed in an analysis of frontal $\mathrm{WMH}$ related to regional volumes.

\section{DISCUSSION}

In this study, brain atrophy and the presence of WMHs were more common in participants with TBI compared with healthy controls, but WMH burden was not associated with baseline TBI injury history, military tenure, and age or cognitive, functional, or symptom outcomes. Some regional brain volumes were significantly different between groups as well. Exploratory analyses suggest a potential relationship between the presence of WMH and lower volumes in some brain regions.

This work adds to the growing body of literature about structural MR imaging findings after mTBI, particularly for WMH, which was the most common imaging finding in both mTBI and healthy controls groups. These findings present a clinical challenge, given their nonspecific nature and diverse causative etiologies, including normal aging. We found a greater frequency of frontal lobe involvement in the mTBI group. While this approached significance, the pattern supports the hypothesis that WMH related to non-TBI causes such as chronic small vessel ischemia or normal aging may demonstrate a more widespread distribution.

The types of imaging findings we observed were similar to those of another large military cohort by Riedy et al. ${ }^{9}$ However, we identified WMH in $81.4 \%$ of our mTBI group, compared with $51.8 \%$ in that study, which also included individuals with moderate and severe TBI. We found a $4 \%$ prevalence of microhemorrhage versus $7 \%$ in that study. Methodologic differences could 


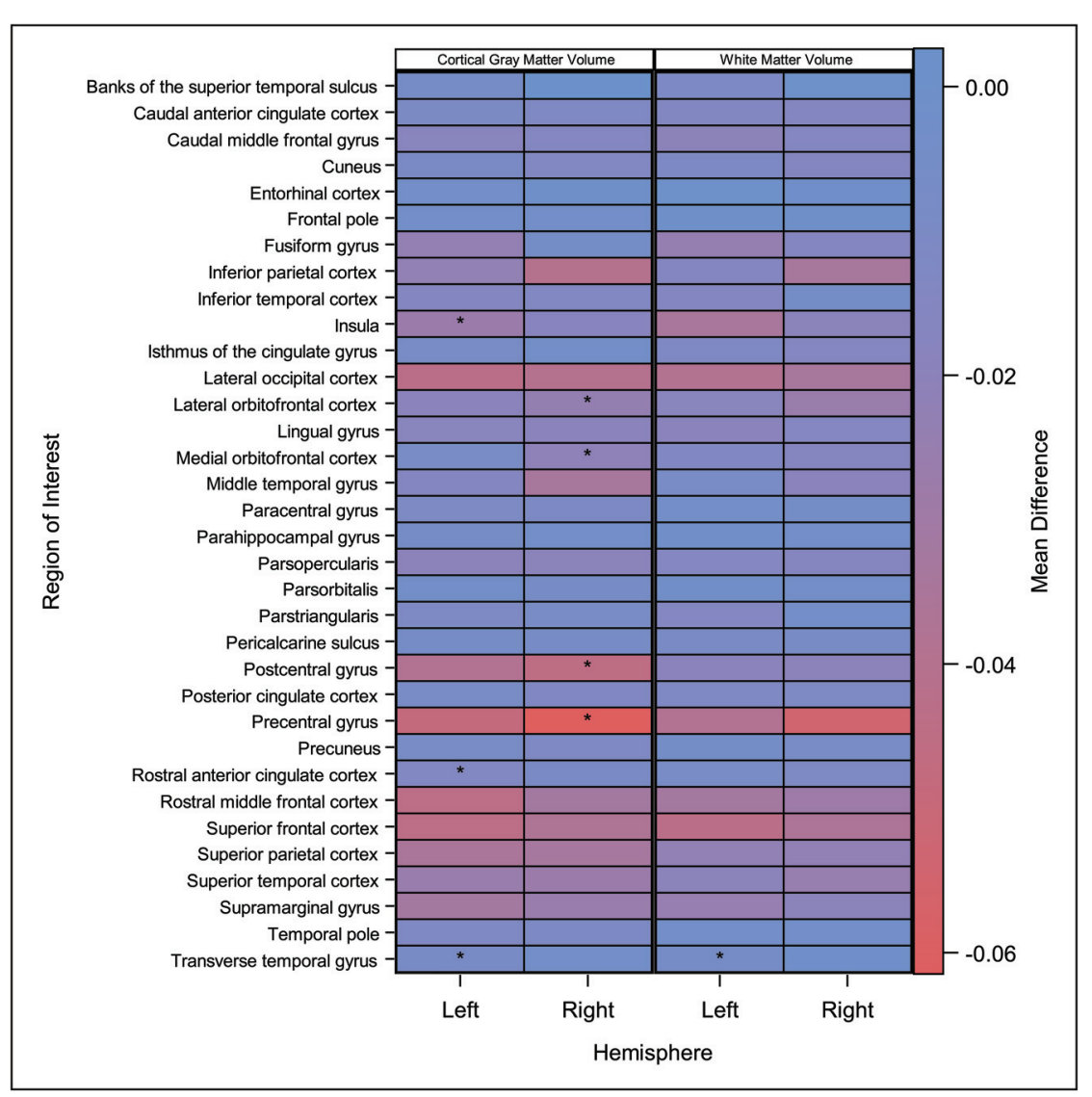

FIG 2. FreeSurfer regional volume differences between $\mathrm{mTBI}$ and healthy control cohorts. The heat map presents mean differences by study population (mTBI versus healthy control) in FreeSurfer regional volumes by region and hemisphere. Colors within each cell represent the magnitude of the mean difference: Blue represents no difference between groups, and red indicates a negative difference (ie, smaller mean volume in $\mathrm{mTBI}$ compared with the healthy control group). Asterisks within each cell indicate regions where a significant difference in volume (after Holm-Bonferroni correction) was identified between study groups according to general linear models adjusted for age.

explain these discrepancies. We used multiple readers and adjudicated interpretations in contrast to a single reader in Riedy et al. Our neuroradiologists reviewed a volumetric T2 FLAIR sequence with $1-\mathrm{mm}^{3}$ voxels in 3 planes. The Riedy et al radiology reader reviewed a high-resolution sagittal T2 FLAIR sequence with 3$\mathrm{mm}$ reconstructions in the axial and coronal planes. Differences in reconstruction parameters may have given our neuroradiologists greater confidence in identifying small lesions. Our decreased prevalence of microhemorrhage may be secondary to the type of hemosiderin-sensitive sequence used in our protocol (2D T2*-weighted) versus the SWI sequence of Reidy et al. SWI is a more sensitive sequence for small, chronic hemorrhages. ${ }^{31}$

Tate et al ${ }^{11}$ performed SWI and FLAIR imaging in 77 military participants with mTBI comparable in age with our population, though their time from injury was shorter (mean $=10.1$ versus 25.6 months). Tate et al found a $40.3 \%$ rate of FLAIR abnormalities, substantially less than the $80 \%$ rate of BIMA. The study of Tate et al analyzed 3-mm-thick routine FLAIR images in contrast to our volumetric acquisition described above. Furthermore, the study of Tate et al used multiple readers for only a small subset
(15 examinations) of their interpretations. The higher baseline WMH rate in our healthy control group (60\%) versus the orthopedic injury (49\%) and posttraumatic stress disorder (29\%) groups of Tate et al further supports an underlying methodologic difference possibly accounting for the varying rates.

We also observed a high rate of pineal gland cysts in both $\mathrm{mTBI}$ $(46 \%)$ and healthy control groups (44\%). Review of the recent literature demonstrates a high prevalence of pineal cysts in both pediatric ${ }^{32}$ (57\%) and adult populations ${ }^{33}$ (35.1\%). Given the similar rates we observed across study cohorts, we suspect that this finding may be due to improved visualization secondary to higher resolution.

Whole-brain and regional volume loss following moderate and severe TBI is well-documented, but volume loss following mTBI is not well-established. Small sample sizes typically limit such studies (Dean et al, ${ }^{34} n=16$; Spitz et al, ${ }^{35} n=8$; Zhou et al, ${ }^{13} n=28$; Burrowes et $\mathrm{al}^{36}{ }^{36} n$ ) ). Our larger sample size allows our results to further substantiate the relationship between mTBI and regional volume loss, though region-level comparisons with other work are limited by heterogeneous definitions of mTBI, methodology, and varying time to injury.

Our cross-sectional analysis revealed 10 regions with significantly smaller volume in the mTBI versus healthy controls. Future work could explore mTBI symptoms based on the degrees of specific regional volume loss such as in a report by Epstein et $\mathrm{al}^{37}(n=55)$, who found increased symptoms of aggression, depression, and anxiety in subjects with chronic mTBI. They found cortical thinning of the right lateral orbitofrontal cortex; however, findings were not significant after multiple-comparison correction. We observed an analogous significant (adjusted) decrease in the cortical volume of the right lateral and medial orbitofrontal cortices.

Strengths of our study include a single, prospective, standardized imaging and interpretation protocol across mTBI and healthy control groups; and 3 independent neuroradiologists with a priori rules for adjudication and the use of a 3T scanner with a 32-channel head coil. We attempted to minimize interpretation variability and maximize sensitivity for the detection of small lesions using standardized interpretation forms, isotropic multiplanar reconstructions, adjudicated interpretations, and pre-established guidelines for classifying imaging findings. Despite our attempt to establish a robust control group, there were some differences in age and sex between the study cohorts. 
We adjusted for age in statistical models testing between-group differences. Although we were unable to adjust for sex given that only 1 woman enrolled in the mTBI cohort, we conducted a sensitivity analysis excluding women and obtained similar results.

Our mTBI group also had variability in the time since injury and diverse injury types, and this heterogeneity in injury could mask imaging differences between study groups. Another limitation is the lack of preinjury or acute postinjury imaging in our participants, which limits our ability to understand the causative association between mTBI and WMH. Predeployment imaging in military populations may help characterize the chronicity of white matter changes following mTBI. In addition, MR imaging findings, including $\mathrm{WMH}$, have not yet been associated with long-term clinical outcomes after mTBI. Our study supports WMH being more frequent in those with $\mathrm{mTBI}$, but at least in our cohort, WMH burden could not be used to make statements about clinical outcome, which may require a much larger sample size.

\section{CONCLUSIONS}

Group analyses demonstrated statistically significant decreases in regional brain volumes. While group-based differences are of limited value to a radiologist interpreting an individual MR imaging, such findings motivate future effort in establishing volumebased biomarkers for prognostication. We identified structural findings in both mTBI and healthy control cohorts. Because high-resolution scans (3D sequences with isotropic $1-\mathrm{mm}^{3}$ voxels) have become commonly performed for multiple brain-related problems such as traumatic brain injury, stroke, anoxia, inflammation, and dementia, understanding the frequency of imaging findings relative to control groups is crucial.

We found a higher prevalence of WMH burden than Riedy et $\mathrm{al}^{9}$ and Tate et al. ${ }^{11}$ While methodologic differences could account for this prevalence, our results emphasize the importance of a dedicated mTBI imaging protocol such as the Defense Centers of Excellence for Psychological Health and Traumatic Brain Injury with high-resolution structural imaging (https:// health.mil/Reference-Center/Forms/2013/07/08/Neuroimagingfollowing-TBI-in-non-deployed-setting).

While white matter findings are nonspecific and a clinical challenge, we observed a greater prevalence of $\geq 1 \mathrm{WMH}$ in our mTBI-versus-healthy control groups after adjustment for age. A frontal-only distribution of WMH was observed more commonly in the mTBI group. Our results suggest that prior trauma should be considered in the differential diagnosis of multifocal white matter abnormalities with a clinical history suggesting even mTBI.

\section{ACKNOWLEDGMENTS}

The authors thank Thomas Perkins, $\mathrm{PhD}$, for design/implementation of the MR imaging protocol; Pavel Krapiva, MD, for image interpretation (with author J.B.P.); Kayla Deru for manuscript review; and the BIMA/NORMAL study team who made this multidisciplinary effort possible. They also acknowledge the instrumental contributions of William Orrison Jr, MD, for BIMA/NORMAL neuroimaging protocol design and image interpretation. Dr Orrison died on October 19, 2017.

Disclosures: Jigar B. Patel—UNRELATED: Other: Lovelace Biomedical Research, Albuquerque, New Mexico, Comments: For a portion of the study described in this article, I was a paid subject matter expert for a research firm that was a subcontractor to the "prime" awardee for this work. The remaining portion of the time, I worked as a volunteer. There is no conflict of interest with the subject matter, and this affiliation is listed on the title page of the article. Steffanie H. Wilson-RELATED: Fees for Participation on Review Activities Such as Data Monitoring Boards, Statistical Analysis, End Point Committees, and the Like: United States Army Medical Research and Materiel Command, Comments: This work was supported by the United States Army Medical Research and Materiel Command Contract No. W81XWH-15-D-0039-0003*; Payment for Writing or Reviewing the Manuscript: US Army Medical Research and Materiel Command, Comments: This work was supported by the United States Army Medical Research and Materiel Command Contract No. W81XWH-15-D-0039-0003.* Terrence R. Oakes-UNRELATED: Consulting Fee or Honorarium: Lovelace Respiratory Research Institute; Payment for Writing or Reviewing the Manuscript: Lovelace Respiratory Research Institute. Lindell K. WeaverRELATED: Grant: United States Army Medical Research and Materiel Command, Comments: grant paid to my institution for all principal investigator activities associated with this work (protocol development, study oversight, travel, and manuscript writing and review).* *Money paid to institution.

\section{REFERENCES}

1. Taylor CA, Bell JM, Breiding MJ, et al. Traumatic Brain InjuryRelated Emergency Department Visits, Hospitalizations, and Deaths: United States, 2007 and 2013. MMWR Surveill Summ 2017; 66:1-16 CrossRef Medline

2. McMahon P, Hricik A, Yue JK, et al. Symptomatology and functional outcome in mild traumatic brain injury: results from the prospective TRACK-TBI study. J Neurotrauma 2014;31:2633 CrossRef Medline

3. Nelson LD, Temkin NR, Dikmen S, et al. Recovery after mild traumatic brain injury in patients presenting to US Level I trauma centers: A Transforming Research and Clinical Knowledge in Traumatic Brain Injury (TRACK-TBI) study. JAMA Neurol 2019; Jun 3. [Epub ahead of print] CrossRef Medline

4. Management of Concussion-mild Traumatic Brain Injury Working Group. VA/DoD Clinical Practice Guideline for Management of Concussion/Mild Traumatic Brain Injury. Washington, DC: Department of Veterans Affairs, Department of Defense; 2016

5. Shetty VS, Reis MN, Aulino JM, et al. ACR Appropriateness Criteria Head Trauma. J Am Coll Radiology 2016;13:668-79 CrossRef Medline

6. Wu X, Kirov II, Gonen O, et al. MR imaging applications in mild traumatic brain injury: an imaging update. Radiology 2016;279:693-707 CrossRef Medline

7. DCoE Clinical Recommendation. Neuroimaging following Mild Traumatic Brain Injury in the Non-Deployed Setting. July 2013. https:/health.mil/Reference-Center/Forms/2013/07/08/Neuroimagingfollowing-TBI-in-non-deployed-setting. Accessed March 25, 2019

8. Reneman L, de Win MM, Booij J, et al. Incidental head and neck findings on MRI in young healthy volunteers: prevalence and clinical implications. AJNR Am J Neuroradiol 2012;33:1971-74 CrossRef Medline

9. Riedy G, Senseney JS, Liu W, et al. Findings from structural MR imaging in military traumatic brain injury. Radiology 2016;279:207-15 CrossRef Medline

10. Orrison WW, Hanson EH, Alamo T, et al. Traumatic brain injury: a review and high-field MRI findings in $\mathbf{1 0 0}$ unarmed combatants using a literature-based checklist approach. J Neurotrauma 2009;26:689-701 CrossRef Medline

11. Tate DF, Gusman M, Kini J, et al. Susceptibility weighted imaging and white matter abnormality findings in service members with 
persistent cognitive symptoms following mild traumatic brain injury. Mil Med 2017;182:e1651-58 CrossRef Medline

12. Fischl B, Salat DH, Busa E, et al. Whole brain segmentation: automated labeling of neuroanatomical structures in the human brain. Neuron 2002;33:341-55 CrossRef Medline

13. Zhou Y, Kierans A, Kenul D, et al. Mild traumatic brain injury: longitudinal regional brain volume changes. Radiology 2013;267:88090 CrossRef Medline

14. Weaver LK, Wilson SH, Lindblad AS, et al. Hyperbaric oxygen for post-concussive symptoms in United States military service members: a randomized clinical trial. Undersea Hyperb Med 2018;45:129-56 Medline

15. Weaver LK, Wilson SH, Lindblad AS, et al; NORMAL Study Team. Comprehensive evaluation of healthy volunteers using multi-modality brain injury assessments: an exploratory, observational study. Front Neurol 2018;9:1030 CrossRef Medline

16. Corrigan JD, Bogner J. Initial reliability and validity of the Ohio State University TBI Identification Method. I Head Trauma Rehabil 2007;22:318-29 CrossRef Medline

17. Weaver LK, Chhoeu AH, Lindblad AS, et al. Hyperbaric oxygen for mild traumatic brain injury: design and baseline summary. Undersea Hyperb Med 2016;43:489-508 Medline

18. Rosset A, Spadola L, Ratib O. OsiriX: an open-source software for navigating in multidimensional DICOM images. J Digit Imaging 2004;17:205-16 CrossRef Medline

19. Grinnon ST, Miller K, Marler JR, et al. National Institute of Neurological Disorders and Stroke Common Data Element Project: approach and methods. Clin Trials 2012;9:322-29 CrossRef Medline

20. Brandstack N, Kurki T, Tenovuo O, et al. MR imaging of head trauma: visibility of contusions and other intraparenchymal injuries in early and late stage. Brain Inj 2006;20:409-16 CrossRef Medline

21. Segonne F, Dale AM, Busa E, et al. A hybrid approach to the skull stripping problem in MRI. Neuroimage 2004;22:1060-75 CrossRef Medline

22. Fischl B, Salat DH, van der Kouwe AJ, et al. Sequence-independent segmentation of magnetic resonance images. Neuroimage 2004;23 (Suppl 1):S69-84 CrossRef Medline

23. Sled JG, Zijdenbos AP, Evans AC. A nonparametric method for automatic correction of intensity nonuniformity in MRI data. IEEE Trans Med Imaging 1998;17:87-97 CrossRef Medline

24. Fischl B, Liu A, Dale AM. Automated manifold surgery: constructing geometrically accurate and topologically correct models of the human cerebral cortex. IEEE Trans Med Imaging 2001;20:70-80 CrossRef Medline
25. Segonne F, Pacheco J, Fischl B. Geometrically accurate topologycorrection of cortical surfaces using nonseparating loops. IEEE Trans Med Imaging 2007;26:518-29 CrossRef Medline

26. Dale AM, Fischl B, Sereno MI. Cortical surface-based analysis, I: segmentation and surface reconstruction. Neuroimage 1999;9:17994 CrossRef Medline

27. Dale AM, Sereno MI. Improved localization of cortical activity by combining EEG and MEG with MRI cortical surface reconstruction: a linear approach. J Cogn Neurosci 1993;5:162-76 CrossRef Medline

28. Fischl B, Dale AM. Measuring the thickness of the human cerebral cortex from magnetic resonance images. Proc Natl Acad Sci U S A 2000;97:11050-55 CrossRef Medline

29. Holm S. A simple sequentially rejective multiple test procedure. Scand J Stat 1979;6:65-70

30. Weaver LK, Churchill S, Wilson SH, et al. A composite outcome for mild traumatic brain injury in trials of hyperbaric oxygen. Undersea Hyperb Med 2019;46:341-52 Medline

31. Akiyama Y, Miyata K, Harada K, et al. Susceptibility-weighted magnetic resonance imaging for the detection of cerebral microhemorrhage in patients with traumatic brain injury. Neurol Med Chir (Tokyo) 2009;49:97-99; discussion 99 CrossRef Medline

32. Bumb JM, Brockmann MA, Groden C, et al. TrueFISP of the pediatric pineal gland: volumetric and microstructural analysis. Clin Neuroradiol 2012;22:69-77 CrossRef Medline

33. Nolte I, Brockmann MA, Gerigk L, et al. TrueFISP imaging of the pineal gland: more cysts and more abnormalities. Clin Neurol Neurosurg 2010;112:204-08 CrossRef Medline

34. Dean PJ, Sato JR, Vieira G, et al. Long-term structural changes after mTBI and their relation to post-concussion symptoms. Brain Inj 2015;29:1211-18 CrossRef Medline

35. Spitz G, Bigler ED, Abildskov T, et al. Regional cortical volume and cognitive functioning following traumatic brain injury. Brain $\operatorname{Cogn}$ 2013;83:34-44 CrossRef Medline

36. Burrowes SA, Rhodes CS, Meeker TJ, et al. Decreased grey matter volume in mTBI patients with post-traumatic headache compared to headache-free mTBI patients and healthy controls: a longitudinal MRI study. Brain Imaging Behav 2019 Apr 12. [Epub ahead of print] CrossRef Medline

37. Epstein DJ, Legarreta M, Bueler E, et al. Orbitofrontal cortical thinning and aggression in mild traumatic brain injury patients. Brain Behav 2016;6:e00581 CrossRef Medline 